\title{
Prognostic value of continuous EEG monitoring during therapeutic hypothermia after cardiac arrest
}

Andrea O Rossetti ${ }^{1+}$, Luis A Urbano ${ }^{2 \dagger}$, Frederik Delodder ${ }^{2}$, Peter W Kaplan ${ }^{3}$, Mauro Oddo ${ }^{2 *}$

\begin{abstract}
Introduction: Continuous EEG (CEEG) is increasingly used to monitor brain function in neuro-ICU patients. However, its value in patients with coma after cardiac arrest (CA), particularly in the setting of therapeutic hypothermia $(\mathrm{TH})$, is only beginning to be elucidated. The aim of this study was to examine whether cEEG performed during $\mathrm{TH}$ may predict outcome.
\end{abstract}

Methods: From April 2009 to April 2010, we prospectively studied 34 consecutive comatose patients treated with $\mathrm{TH}$ after CA who were monitored with CEEG, initiated during hypothermia and maintained after rewarming. EEG background reactivity to painful stimulation was tested. We analyzed the association between CEEG findings and neurologic outcome, assessed at 2 months with the Glasgow-Pittsburgh Cerebral Performance Categories (CPC).

Results: Continuous EEG recording was started $12 \pm 6$ hours after CA and lasted $30 \pm 11$ hours. Nonreactive cEEG background (12 of $15(75 \%)$ among nonsurvivors versus none of $19(0)$ survivors; $P<0.001$ ) and prolonged discontinuous "burst-suppression" activity (11 of $15(73 \%)$ versus none of $19 ; P<0.001$ ) were significantly associated with mortality. EEG seizures with absent background reactivity also differed significantly (seven of 15 $(47 \%)$ versus none of $12(0) ; P=0.001)$. In patients with nonreactive background or seizures/epileptiform discharges on CEEG, no improvement was seen after TH. Nonreactive cEEG background during TH had a positive predictive value of $100 \%$ (95\% confidence interval $(\mathrm{Cl}), 74$ to $100 \%)$ and a false-positive rate of $0(95 \% \mathrm{Cl}, 0$ to $18 \%)$ for mortality. All survivors had CEEG background reactivity, and the majority of them (14 (74\%) of 19) had a favorable outcome (CPC 1 or 2).

Conclusions: Continuous EEG monitoring showing a nonreactive or discontinuous background during $\mathrm{TH}$ is strongly associated with unfavorable outcome in patients with coma after CA. These data warrant larger studies to confirm the value of continuous EEG monitoring in predicting prognosis after CA and $\mathrm{TH}$.

\section{Introduction}

Therapeutic hypothermia ( $\mathrm{TH})$ improves outcome in comatose survivors of cardiac arrest (CA) [1-3]. TH also alters the predictive value of neurologic prognostication in patients with postanoxic coma [4]. We and others recently demonstrated that, compared with previous studies performed before the introduction of TH [5], neurologic examination performed at 72 hours may be

\footnotetext{
* Correspondence: mauro.oddo@chuv.ch

+ Contributed equally

${ }^{2}$ Department of Intensive Care Medicine, Lausanne University Hospital and Faculty of Biology and Medicine, BH-08, Rue du Bugnon 46, CHUV, 1011

Lausanne, Switzerland

Full list of author information is available at the end of the article
}

unreliable to predict outcome after CA, and that standard EEG may significantly improve prognostication at this time [6,7].

Continuous EEG monitoring (cEEG) provides important information regarding brain function, particularly in comatose patients $[8,9]$, and is increasingly used to monitor early on-line changes of cerebral electrophysiology at the bedside in critically ill patients. Only a few studies have evaluated the role of cEEG performed during $\mathrm{TH}$ in the early phase of postresuscitation care. These studies, however, either included pediatric populations only [10] or were focused primarily on the prevalence of postanoxic seizures [11]. However, the exact prognostic value of cEEG findings during $\mathrm{TH}$ in patients 
with postanoxic coma has not been investigated. In this prospective study, we sought to examine the relation between cEEG findings during $\mathrm{TH}$ and outcome in comatose survivors of CA. We primarily tested the hypothesis that the type and reactivity of cEEG background during $\mathrm{TH}$ may reliably predict patient prognosis.

\section{Materials and methods \\ Patients}

We prospectively studied consecutive comatose adult patients (older than 16 years) admitted from April 2009 to April 2010 to the medicosurgical intensive care unit (ICU) of the University Hospital of Lausanne, who were treated with $\mathrm{TH}$ after successful resuscitation from CA and were monitored with cEEG, initiated during hypothermia. Approval for the study was obtained by the local Institutional Review Board with waiver of informed consent, because cEEG was part of standard patient care. All patients were resuscitated according to current recommendations [2] and treated with mild $\mathrm{TH}$ to $33^{\circ} \mathrm{C}$ for 24 hours. Therapeutic hypothermia was started immediately after admission to the emergency department and was applied by using a cooling technique combining the administration of intravenous ice-cold fluids and the application of a surface cooling device (Arctic Sun System; Medivance, Louisville, CO, USA), according to the protocol in use in our institution $[6,12]$. Midazolam $(0.1 \mathrm{mg} / \mathrm{kg} / \mathrm{h})$ and fentanyl $(1.5 \mu \mathrm{g} / \mathrm{kg} / \mathrm{h})$ were given for sedation-analgesia, and vecuronium $(0.1 \mathrm{mg} / \mathrm{kg}$ boluses) was administered to control shivering.

\section{Continuous EEG data}

Video-cEEG (Viasys Neurocare, Madison, WI, USA) was started as soon as possible after ICU admission and during $\mathrm{TH}$, by using nine to 21 electrodes arranged according to the international 10-20 system, and was maintained up to at least 6 hours after rewarming. Background reactivity on cEEG was tested with repetitive auditory, visual, and nociceptive stimulations performed by an experienced neurologist during and after $\mathrm{TH}$, as described in our previous study [6]. Within 4 hours after the end of cEEG, all recordings were interpreted by two EEG-certified neurologists; cEEG background reactivity was considered present if cerebral electrical activity of at least $10 \mu \mathrm{V}$ (regardless of frequency range) was observed, and EEG background showed any clear and reproducible change in amplitude or frequency on simulation, excluding "stimulus-induced rhythmic, periodic, or irritative discharges" (SIRPIDS) or induction of muscle artifact alone. Stimulation and EEG background activity were assessed in all patients after at least 12 hours after the start of $\mathrm{TH}$ (that is, during the maintenance phase of $\mathrm{TH}$ ) and within 24 hours from CA: thus, EEG background reactivity was tested before the 72-hour delay recommended by the American Academy of Neurology [5]. EEG background interrupted by flat periods was labeled as "discontinuous" (in this setting, also known as "burst-suppression") if this pattern was found over the whole recording. Repetitive or rhythmic, focal or generalized spikes, sharp waves, spike and waves, or rhythmic waves evolving in amplitude, frequency, or field were categorized as "epileptiform," as detailed in our previous studies $[6,13,14]$.

\section{Additional standard assessments and treatment}

The following investigations were performed shortly after rewarming, at least 36 hours after $\mathrm{CA}$, at a patient core temperature $>35^{\circ} \mathrm{C}$ and off sedation, as previously reported [6]: repeated neurologic examination, a standard (30 minute) EEG with the previously mentioned stimulations, and cortical somatosensory evoked potentials (SSEPs). Patients with EEG evidence of status epilepticus were treated with intravenous antiepileptic drugs (including levetiracetam, midazolam, valproate, or propofol for at least 24 hours), as reported in our previous study [14]. Treatment was discontinued if no clinical improvement was noted after at least 72 hours, together with incomplete recovery of all brainstem reflexes (pupillary, oculocephalic, corneal), and/or bilaterally absent cortical response of SSEPs, in accordance with current recommendations [5]. Physicians were not blinded to the cEEG results; however, cEEG findings were not used to guide therapy or to decide withdrawal of care.

\section{Data collection}

Baseline demographics, including type of CA (ventricular fibrillation (VF) versus non-VF, including asystole and pulseless electrical activity), time from CA to return of spontaneous circulation (ROSC), etiology of CA (cardiac versus noncardiac), and time from CA to temperature target of $33^{\circ} \mathrm{C}$ were prospectively collected. The following cEEG data were recorded during $\mathrm{TH}$ and included in the analysis: presence/absence of background reactivity, presence/absence of discontinuous EEG background, and presence/absence of epileptiform abnormalities.

\section{Outcome assessment}

In-hospital mortality was used as primary outcome. Neurologic outcome was assessed at 2 months by review of the computerized database of our hospital or a phone interview, and categorized according to the Glasgow-Pittsburgh Cerebral Performance Categories (CPC), in which $1=$ good recovery, 2 = moderate disability, $3=$ severe disability with dependency for daily-life activity, 4 = vegetative state, and $5=$ death [15], and outcome was dichotomized as good (CPC 1 and 2) versus poor (CPC 3 to 5 ). 


\section{Statistical analysis}

Quantitative parameters are reported as median and range, and dichotomous variables, as number and percentage. Two-sided $t$ tests, Fisher Exact, and MannWhitney $U$ tests were used as needed. Significance was assumed at a level of $P<0.01$, applying conservative analysis for multiple comparisons between variables (Bonferroni corrections, with five tests). Positive (PPV) and negative (NPV) predictive values for mortality and false-positive rates (FPR; 1-specificity) were calculated by using a binomial $95 \%$ CI. Area under the receiver operating characteristic (ROC) curve was used to assess the predictive values for mortality, and comparisons were analyzed by using nonparametric tests. Calculations were performed with Stata software, version 9 (College Station, TX, USA).

\section{Results}

Patients

We studied 34 comatose CA survivors treated with $\mathrm{TH}$ for 24 hours and monitored with cEEG during TH. Mean patient age was $61 \pm 13$ years; median time from CA to ROSC was 20 (interquartile range, 10 to 30 ) minutes; mean time from CA to cEEG recording was $12 \pm 6$ hours; cEEG lasted a mean of $30 \pm 11$ hours. No complication related to the cEEG was observed; shivering, muscle, or electrode artifacts were transient and did not interfere with interpretation.

Relation between baseline clinical variables and outcome At 2 months, 15 patients died, and 19 patients survived. The majority of survivors (14 (74\%) of 19 patients) had a good outcome ( $n=8$ with CPC $1 ; n=6$ with CPC 2 ), whereas the remaining five patients had CPC 3 . No patient remained in a vegetative state. Baseline demographic variables, including gender, initial arrest rhythm, CA etiology, and time from CA to ROSC were comparable between survivors and nonsurvivors (Table 1).

\section{Early continuous EEG findings and outcome}

Representative examples of EEG recordings during $\mathrm{TH}$ are given in Figure 1, showing one patient with a reactive cEEG background who eventually had a good recovery (Figure 1) and another patient with a persistent discontinuous EEG background activity alternating with generalized, electrical seizures ("seizure-suppression pattern"), who eventually died (Figure 2).

The association between outcome and cEEG findings during $\mathrm{TH}$ is shown in Table 2. After adjusting for multiple comparisons, nonreactive EEG background, persistent discontinuous EEG pattern, and presence of seizures/epileptiform discharges were strongly associated with mortality. Importantly, all patients with epileptiform abnormalities or persistent discontinuous EEG background or both also showed absent EEG reactivity. Predictive values for mortality for these three cEEG features, as well as SSEPs, are shown in Table 3. Despite relatively wide confidence intervals due to the small sample size, the positive predictive value (PPV) was $100 \%$, and the false-positive rate (FPR) was 0 , thus indicating excellent prognostic value for early cEEG features. Of note, compared with patients with a reactive cEEG background, those with nonreactive cEEG backgrounds received similar weight-adjusted doses of midazolam $(P=0.49 ; t$ test $)$ and fentanyl $(P=0.33$; $t$ test $)$.

\section{Association between outcome and neurologic and electrophysiological examinations at $\mathbf{7 2}$ hours}

Neurologic examination and SSEPs were performed at 72 hours in normothermic conditions, as per protocol at our institution and according to actual recommendations [5]. All nonsurvivors with absent cEEG reactive background during $\mathrm{TH}$ also had absent SSEPs at 72 hours. Although the PPV for mortality of absent cEEGreactive background and bilaterally absent SSEPs was 1.00, the NPV of cEEG was higher than that of SSEP ( 0.83 versus 0.70 ; Table 3$)$. In addition, when using the area under the ROC curve (Figure 3), cEEG reactivity yielded better prediction than did SSEP, with a statistically significant difference in the predictive ability in favor of EEG background reactivity over SSEPs $(0.88$ versus $0.69 ; P=0.006$ ).

Incomplete recovery of brainstem reflexes (pupillary, oculocephalic, corneal) and absent or extension motor reaction to pain also differed among survivors and nonsurvivors (three of 19 versus 11 of 15 , and three of 19 versus 15 of 15, respectively); however, the false-positive rate was greater than zero for both, confirming that

Table 1 Patient baseline characteristics in survivors versus nonsurvivors

\begin{tabular}{lll}
\hline & Survivors $(\boldsymbol{n}=\mathbf{1 9})$ & Nonsurvivors $(\boldsymbol{n}=\mathbf{1 5})$ \\
\hline Female gender, number (\%) & $6(32 \%)$ & $3(20 \%)$ \\
Median age, years (range) & $62(35-84)$ & $64(32-73)$ \\
Initial CA rhythm ventricular fibrillation, number (\%) & $14(73 \%)$ & $10(67 \%)$ \\
CA of cardiac etiology, number (\%) & $16(84 \%)$ & $11(73 \%)$ \\
Median time from CA to ROSC, minutes (range) & $20(5-40)$ & $22(8-180)$ \\
\hline
\end{tabular}

$\mathrm{CA}$, cardiac arrest; ROSC, return of spontaneous circulation. 


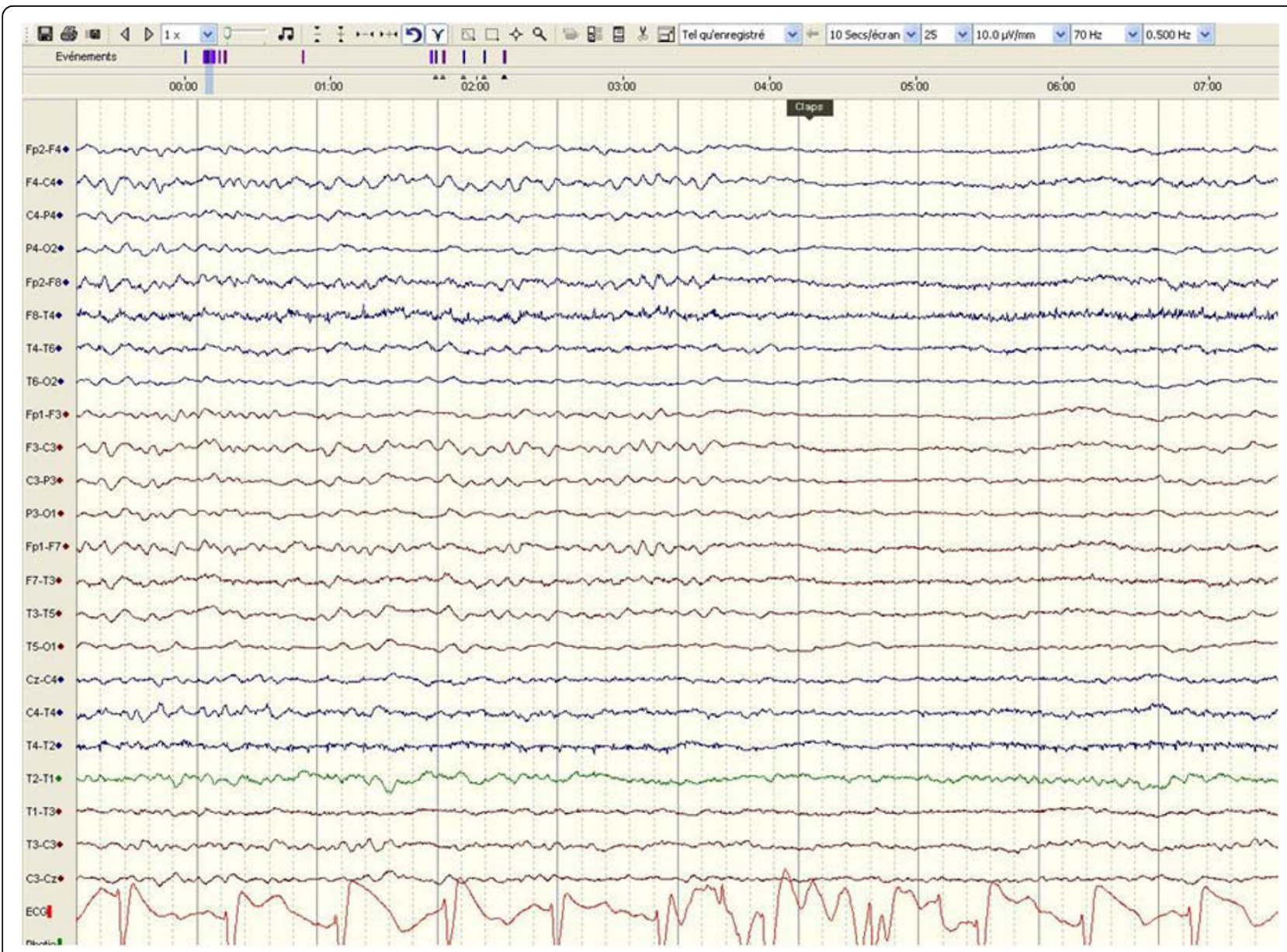

Figure 1 EEG recording performed during therapeutic hypothermia from one representative patient who had a good outcome (Cerebral Performance Category 1 at $\mathbf{2}$ months). EEG shows a reactive EEG background activity to sound ("claps"); recording, $30 \mathrm{~mm} / \mathrm{sec}, 10$ $\mu \mathrm{V} / \mathrm{mm}$.

neurologic examination alone may not be reliable in predicting the outcome after $\mathrm{CA}$ and $\mathrm{TH}$.

\section{Postanoxic seizures and epileptiform discharges}

The total number of patients with epileptiform EEG features during the entire study period was eight (26\%) of 34. Five had generalized electrographic seizures alternating with diffuse suppression ("seizure-suppression" pattern), and two had generalized, sustained periodic epileptiform discharges (G-PEDs), again alternating with generalized background suppressions. One patient had delayed seizures that became apparent only after $\mathrm{TH}$ and rewarming. None of the seven patients with early (that is, during $\mathrm{TH}$ ) epileptiform abnormalities showed a significant improvement on the standard EEG performed after $\mathrm{TH}$ in normothermic conditions. Furthermore, all had a nonreactive EEG background and died.

In contrast, in the single patient with delayed (that is, after $\mathrm{TH}$, at normothermia) postanoxic seizures, cEEG became diffusely epileptiform with multifocal myoclonia only after weaning of sedation: of note, cEEG background remained reactive despite epileptiform activity, and the patient regained consciousness and survived.

\section{Discussion}

The main results of this single-center prospective study can be summarized as follows: (1) absent EEG background reactivity observed during the maintenance phase of $\mathrm{TH}$ appeared to be strongly associated with poor outcome in patients with coma after CA; (2) all patients in whom cEEG showed background reactivity to painful stimuli survived, and the large majority (74\%) awoke and had a favorable outcome; (3) persistent discontinuous background and the presence of seizures or epileptiform discharges on cEEG were also strong risk factors for poor outcome; (4) nonreactive cEEG background yielded a significantly better prognostic value than SSEPs, mostly because of a higher negative predictive value; (5) EEG reactivity to painful stimulation did not seem to be affected by $\mathrm{TH}$, because all patients with 


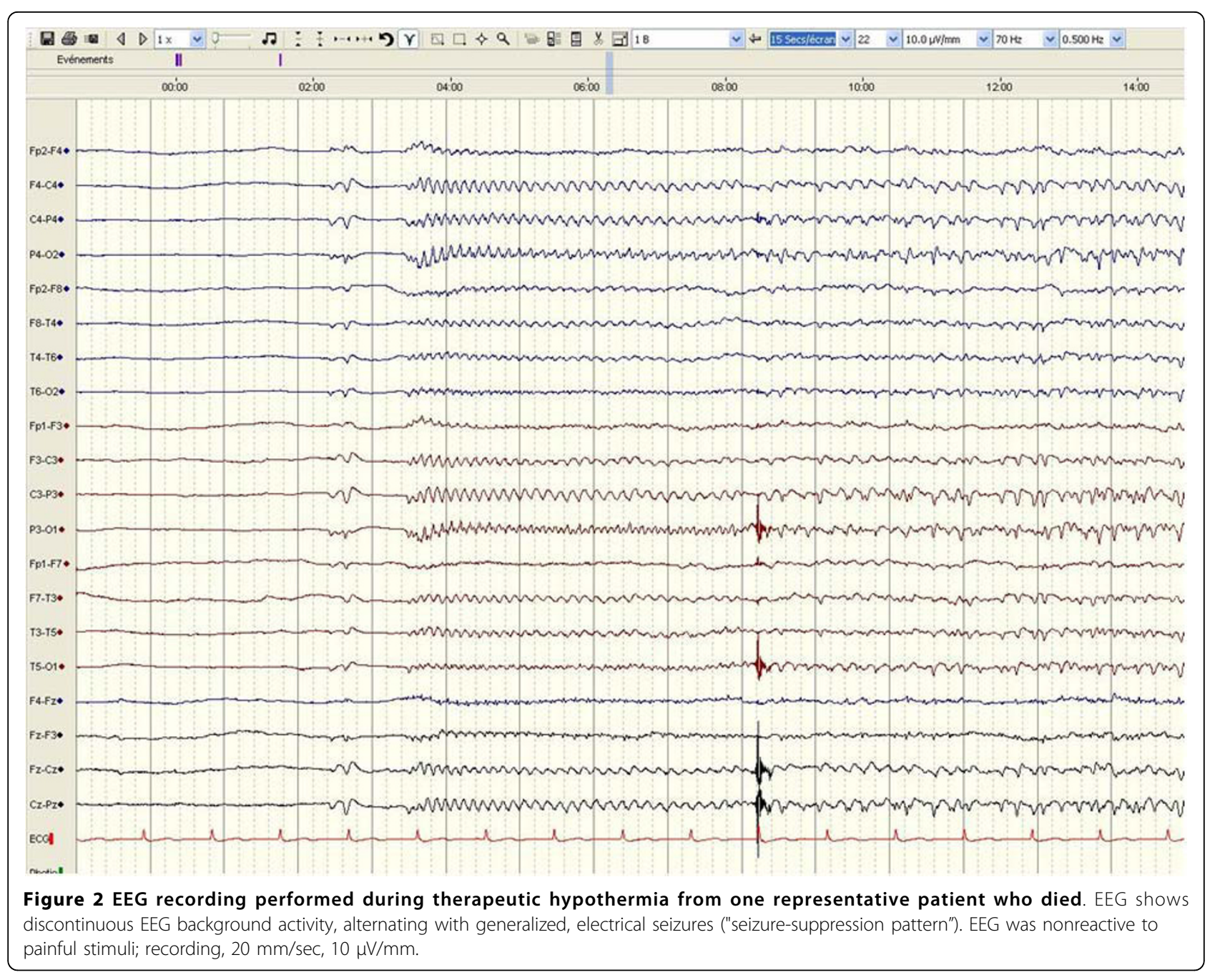

absent background reactivity during $\mathrm{TH}$ had similar findings on the EEG performed in normothermic conditions, and it was not influenced by sedation-analgesia.

To our knowledge, this is the first clinical study showing that nonreactive EEG background activity during $\mathrm{TH}$ is an early predictor of poor outcome in patients with postanoxic coma. Before $\mathrm{TH}$ became a widely used treatment of hypoxic/ischemic encephalopathy, diffuse EEG background suppression below $20 \mu \mathrm{V}$, burst-suppression with generalized epileptiform activity, or generalized periodic complexes on a flat background have been associated with poor outcome $[16,17]$. This was recently confirmed by our group in patients treated with $\mathrm{TH}$, in whom standard EEG was performed at the end of treatment in normothermic conditions [6]. Moreover, prolonged epileptiform EEG features are independently correlated with mortality after postanoxic coma [13], in patients assessed both after [6] and during $[10,13] \mathrm{TH}$. However, none of these studies formally addressed the predictive value of any of the EEG

Table 2 Continuous EEG characteristics in survivors versus nonsurvivors

\begin{tabular}{llll}
\hline & Survivors $(\boldsymbol{n}=\mathbf{1 9})$ & Nonsurvivors $(\boldsymbol{n}=\mathbf{1 5})$ & $\boldsymbol{P}$ value (test) \\
\hline Time from CA to initiation of cEEG, hours (range) & $16(3-23)$ & $10(1-21)$ & $0.11(\mathrm{U})$ \\
Median CEEG duration, hours (range) & $26(19-48)$ & $26(22-66)$ & $0.17(\mathrm{U})$ \\
Nonreactive CEEG background, number (\%) & $0(0)$ & $12(75 \%)$ & $<0.001($ Fisher) \\
Prolonged discontinuous activity ("burst-suppression"), number (\%) & $0(0)$ & $11(73 \%)$ & $<0.001$ (Fisher) \\
EEG seizures or epileptiform discharges, number (\%) & $0(0)$ & $7(47 \%)$ & 0.001 (Fisher) \\
\hline
\end{tabular}

$\mathrm{CA}$, cardiac arrest. 
Table 3 Prognostic predictive value of continuous EEG (30-day mortality)

\begin{tabular}{llll}
\hline & PPV & NPV & FPR \\
\hline Nonreactive background & $1.00(0.74-1.00)$ & $0.83(0.65-0.97)$ & $0(0-0.18)$ \\
Prolonged discontinuous activity ("burst-suppression") & $1.00(0.71-1.00)$ & $0.86(0.61-0.95)$ & $0(0-0.18)$ \\
Seizures/epileptiform discharges & $1.00(0.59-1.00)$ & $0.70(0.50-0.86)$ & $0(0-0.18)$ \\
Bilaterally absent SSEPs & $1.00(0.48-1.00)$ & $0.70(0.50-0.86)$ & $0(0-0.18)$ \\
\hline
\end{tabular}

FPR, false-positive rate; NPV, negative predictive value; PPV, positive predictive value; SSEPs, somatosensory evoked potentials.

findings during $\mathrm{TH}$ or compared the value of EEG with that of neurologic examination or SSEPs, the latter being regarded as reliable predictors of poor prognosis [5]. We have recently shown that background reactivity performed after $\mathrm{TH}$ in normothermic conditions is a strong outcome predictor of postanoxic coma [6], and thus undertook this study to examine the prognostic value of EEG background performed during $\mathrm{TH}$ in the early phase after CA. Our present findings confirm our previous study and indeed seem to suggest that reactive background on cEEG has a strong prognostic predictive value, even when monitoring is performed during $\mathrm{TH}$. They also suggest that background reactivity is not significantly influenced by core temperature or by sedation. After earlier reports on favorable outcome for patients showing continuous amplitude-integrated EEG after TH [18], a recent study on 30 patients showed that quantitative EEG features during TH (burst-suppression ratio, response entropy, state entropy) were significantly associated with long-term functional outcome [19].
Although our results are in line with these findings, we add important concomitant clinical information and describe a much easier approach for EEG interpretation, without the need for more-complicated and not easily available software analysis.

Although our study was not primarily focused on the epidemiology of postanoxic seizures, this issue deserves further discussion. Previous studies reported a variable prevalence of postanoxic seizures from 10\% [11] to $47 \%$ [10]. We observed a $21 \%$ prevalence (seven of 34 patients) of epileptiform abnormalities during $\mathrm{TH}$, of whom five patients ( $15 \%$ of the entire cohort) had sustained EEG seizures. Because mild hypothermia and sedation (midazolam in our study) have antiepileptic action, the occurrence of electrical seizures during $\mathrm{TH}$ may reflect more-severe and diffuse brain injury. This might explain why none of the seven patients with seizures during $\mathrm{TH}$ survived, in line with previous observations [11]. In contrast, it appears that seizures occurring only at the end of $\mathrm{TH}$, after rewarming and off sedation,

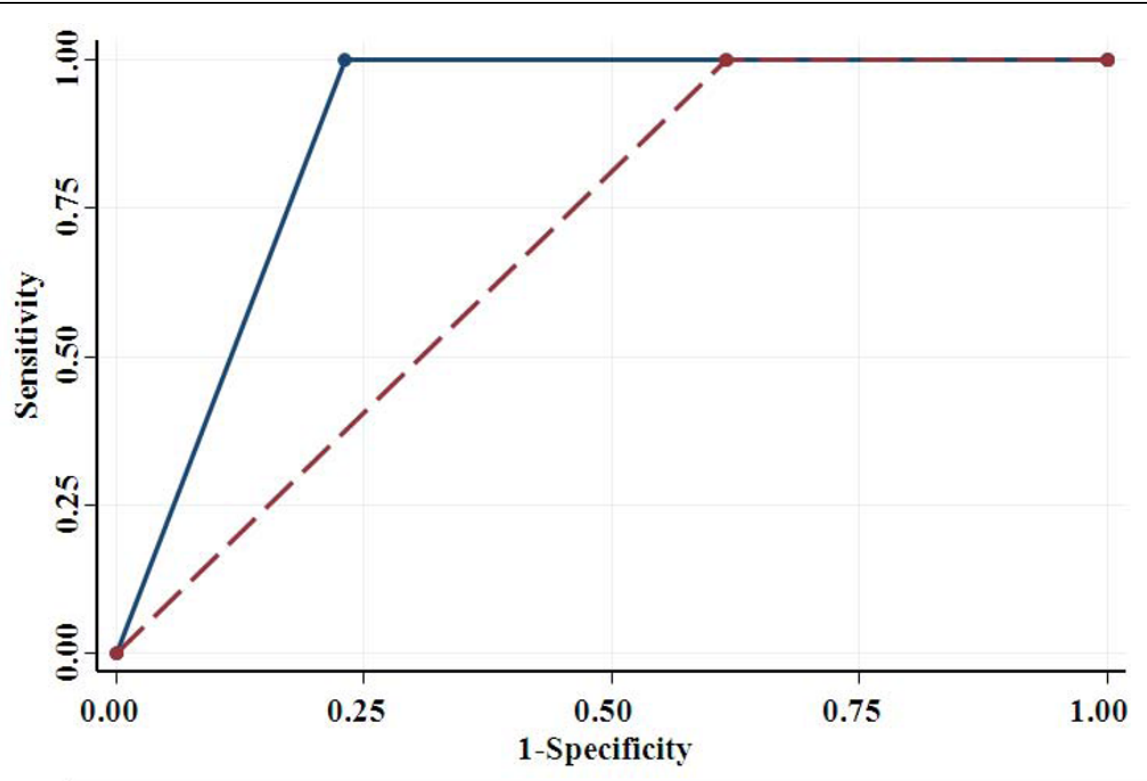

EEGreact ROC area 0.89

$\longrightarrow \longrightarrow-$ SSEP ROC area: 0.69

Figure 3 Area under the receiver operating characteristic (ROC) curve for mortality prediction of cEEG reactivity (performed during therapeutic hypothermia, blue line) and of somatosensory evoked potentials (SSEPs, performed in normothermic conditions, red line). Continuous EEG yielded better prediction than SSEPS (ROC area, 0.88 versus $0.69 ; P=0.006$ ). 
carry a better prognosis, possibly because brain injury is less severe (thus they are effectively treated with induced hypothermia and sedatives). Indeed, one patient in our cohort, treated for status epilepticus that developed after $\mathrm{TH}$, survived. Altogether, these data underline the value of early cEEG for the treatment of comatose CA patients treated with $\mathrm{TH}$.

\section{Study limitations}

This study has several limitations. First, the sample size is limited; thus our results are to be considered preliminary and will need further confirmation by other groups and larger studies. However, for this reason, we applied conservative statistical corrections for multiple comparisons (Bonferroni). Second, it was a single-center study, thus data cannot be generalized. Some subjectivity may also be related to the scoring of EEG reactivity; however, we used the same method described in our recent report, which included more than 100 patients. Time from CA to initiation of cEEG did not differ significantly between survivors and nonsurvivors (Table 2); thus it is unlikely that timing of cEEG affected the predictive value of the test. Finally, because the cEEG was interpreted before knowing final patient prognosis, it is unlikely that it influenced outcome. Furthermore, although clinicians were aware of cEEG results, EEG findings (both during $\mathrm{TH}$ and at normothermia) were not used to guide therapy or decisions for withdrawal of care; thus we believe that this contributed to minimize the so-called "self-fulfilling prophecy" phenomenon [6].

\section{Conclusions}

Continuous EEG background abnormalities during TH seem to be strongly associated with outcome after CA and appear to yield excellent point estimates for positive predictive values and false-positive rates for mortality. Our data suggest that continuous EEG may be of value in predicting outcome after $\mathrm{CA}$ and $\mathrm{TH}$. Additional larger prospective studies are needed to confirm our findings and to verify further whether continuous EEG can be helpful for the prognostic assessment of postanoxic coma.

\section{Key messages}

- The results of this single-center study show that the presence of background reactivity on continuous EEG monitoring (cEEG) performed during therapeutic hypothermia is associated with 30-day survival and favorable neurologic outcome after cardiac arrest.

- Our preliminary data suggest that nonreactive EEG background carries a dismal outcome and is $100 \%$ predictive of mortality in comatose cardiac-arrest patients.
- Early cEEG findings appear to have a significantly better predictive value than somatosensory evoked potentials performed after $\mathrm{TH}$.

- Additional larger prospective studies are needed to confirm whether continuous EEG may be a helpful tool for the prognostic assessment of postanoxic coma.

\section{Abbreviations}

CA: cardiac arrest; cEEG: continuous electroencephalography; CPC: GlasgowPittsburgh Cerebral Performance Categories; EEG: electroencephalography; FPR: false-positive rate; G-PEDS: generalized, sustained periodic epileptiform discharges; ICU: intensive care unit; NPV: negative predictive value; PPV: positive predictive value; ROC: receiver operating characteristic; ROSC: return of spontaneous circulation; SIRPIDS: stimulus induced rhythmic, periodic, or irritative discharges; SSEPs: somatosensory evoked potentials; TH: therapeutic hypothermia; VF: ventricular fibrillation.

\section{Acknowledgements}

This study was supported by departmental funding from the Service de Médecine Intensive Adulte and the Département des Neurosciences Cliniques, Centre Hospitalier Universitaire Vaudois (CHUV), University Hospital, Lausanne, Switzerland.

The authors thank Malin Maeder-Ingvar, MD, for her help in the data collection and express their gratitude to all ICU fellows, residents, and nurses, as well as to all EEG technicians for their valuable help.

\section{Author details}

'Department of Clinical Neurosciences, Lausanne University Hospital and Faculty of Biology and Medicine, BH-07, Rue du Bugnon 46, CHUV, 1011 Lausanne, Switzerland. ${ }^{2}$ Department of Intensive Care Medicine, Lausanne University Hospital and Faculty of Biology and Medicine, BH-08, Rue du Bugnon 46, CHUV, 1011 Lausanne, Switzerland. ${ }^{3}$ Department of Neurology, Johns Hopkins Bayview Medical Center, 4940 Eastern Avenue, Baltimore, Maryland 21224, USA.

\section{Authors' contributions}

AOR conceived the study, collected the data, carried out part of the data analysis, and drafted the manuscript. LAU carried out part of the data analysis and drafted the manuscript. FD helped with data collection and study coordination and revised the manuscript. PWK revised the manuscript and gave important intellectual contributions. MO conceived the study, was responsible for study coordination, and revised and helped to draft the manuscript.

\section{Competing interests}

The authors declare that they have no competing interests.

Received: 27 March 2010 Revised: 24 June 2010

Accepted: 29 September 2010 Published: 29 September 2010

\section{References}

1. Hypothermia after Cardiac Arrest Study Group: Mild therapeutic hypothermia to improve the neurologic outcome after cardiac arrest. $N$ Engl J Med 2002, 346:549-556.

2. ECC Committee, Subcommittees and Task Forces of the American Heart Association: American Heart Association Guidelines for Cardiopulmonary Resuscitation and Emergency Cardiovascular Care. Circulation 2005, 112: IV1-IV203.

3. Bernard SA, Gray TW, Buist MD, Jones BM, Silvester W, Gutteridge G, Smith K: Treatment of comatose survivors of out-of-hospital cardiac arrest with induced hypothermia. N Engl J Med 2002, 346:557-563.

4. Young GB: Clinical practice: neurologic prognosis after cardiac arrest. $N$ Engl J Med 2009, 361:605-611.

5. Wijdicks EF, Hijdra A, Young GB, Bassetti CL, Wiebe S: Practice parameter: prediction of outcome in comatose survivors after cardiopulmonary resuscitation (an evidence-based review): report of the Quality 
Standards Subcommittee of the American Academy of Neurology. Neurology 2006, 67:203-210.

6. Rossetti AO, Oddo M, Logroscino G, Kaplan PW: Prognostication after cardiac arrest and hypothermia: a prospective study. Ann Neurol 2010 67:301-307.

7. Al Thenayan E, Savard M, Sharpe M, Norton L, Young B: Predictors of poor neurologic outcome after induced mild hypothermia following cardiac arrest. Neurology 2008, 71:1535-1537.

8. Friedman D, Claassen J, Hirsch LJ: Continuous electroencephalogram monitoring in the intensive care unit. Anesth Analg 2009, 109:506-523.

9. Rossetti AO, Oddo M: The neuro-ICU patient and electroencephalography paroxysms: if and when to treat. Curr Opin Crit Care 2010, 16:105-109.

10. Abend NS, Topjian A, Ichord R, Herman ST, Helfaer M, Donnelly M, Nadkarni V, Dlugos DJ, Clancy RR: Electroencephalographic monitoring during hypothermia after pediatric cardiac arrest. Neurology 2009, 72:1931-1940.

11. Legriel S, Bruneel F, Sediri H, Hilly J, Abbosh N, Lagarrigue MH, Troche G, Guezennec P, Pico F, Bedos JP: Early EEG monitoring for detecting postanoxic status epilepticus during therapeutic hypothermia: a pilot study. Neurocrit Care 2009, 11:338-344.

12. Oddo M, Ribordy V, Feihl F, Rossetti AO, Schaller MD, Chiolero R, Liaudet L: Early predictors of outcome in comatose survivors of ventricular fibrillation and non-ventricular fibrillation cardiac arrest treated with hypothermia: a prospective study. Crit Care Med 2008, 36:2296-2301.

13. Rossetti AO, Logroscino G, Liaudet L, Ruffieux C, Ribordy V, Schaller MD, Despland PA, Oddo M: Status epilepticus: an independent outcome predictor after cerebral anoxia. Neurology 2007, 69:255-260.

14. Rossetti AO, Oddo M, Liaudet L, Kaplan PW: Predictors of awakening from postanoxic status epilepticus after therapeutic hypothermia. Neurology 2009, 72:744-749.

15. Booth CM, Boone RH, Tomlinson G, Detsky AS: Is this patient dead, vegetative, or severely neurologically impaired? Assessing outcome for comatose survivors of cardiac arrest. JAMA 2004, 291:870-879.

16. Bassetti C, Bomio F, Mathis J, Hess CW: Early prognosis in coma after cardiac arrest: a prospective clinical, electrophysiological, and biochemical study of 60 patients. J Neurol Neurosurg Psychiatry 1996, 61:610-615.

17. Zandbergen EG, Hijdra A, Koelman JH, Hart AA, Vos PE, Verbeek MM, de Haan RJ: Prediction of poor outcome within the first 3 days of postanoxic coma. Neurology 2006, 66:62-68.

18. Rundgren M, Rosen I, Friberg H: Amplitude-integrated EEG (aEEG) predicts outcome after cardiac arrest and induced hypothermia. Intensive Care Med 2006, 32:836-842.

19. Wennervirta JE, Ermes MJ, Tiainen SM, Salmi TK, Hynninen MS, Sarkela MO, Hynynen MJ, Stenman UH, Viertio-Oja HE, Saastamoinen KP, Pettilä VY Vakkuri AP: Hypothermia-treated cardiac arrest patients with good neurological outcome differ early in quantitative variables of EEG suppression and epileptiform activity. Crit Care Med 2009, 37:2427-2435.

doi:10.1186/cc9276

Cite this article as: Rossetti et al:: Prognostic value of continuous EEG monitoring during therapeutic hypothermia after cardiac arrest. Critical Care 2010 14:R173.

\section{Submit your next manuscript to BioMed Central and take full advantage of:}

- Convenient online submission

- Thorough peer review

- No space constraints or color figure charges

- Immediate publication on acceptance

- Inclusion in PubMed, CAS, Scopus and Google Scholar

- Research which is freely available for redistribution 